\title{
A Young Girl with Bilateral Wrist Joint Deformity
}

MD. ROBED AMIN, ${ }^{1}$ SAFIN ARARAT RAHMAN, ${ }^{2}$ SHABNAM FATEMA ENAM, ${ }^{2}$ AHMED RIYAD HOSSAIN, ${ }^{3}$ MA KAHHAR ${ }^{4}$

\begin{abstract}
:
Madelung deformity is a rare autosomal disorder involving the epiphyseal plate of distal radial end and consequent deformity. It is primarily found in adolescent females. A teenage girl presented with progressive bilateral wrist joint deformity and bowing of distal end of forearm without significant functional impairment and systemic complaints. Clinical examination was suggestive of Madelung deformity that was confirmed by Radiological evaluation. The pattern of deformity and radiology is the key to identify the disease.
\end{abstract}

Keyword: Wrist Joint, Deformity, Bilateral, Madelung

\section{Introduction:}

Madelung deformity was first described in 1878 by Otto Wilhelm Madelung, German surgeon (1846 - 1926). Madelung deformity should not be confused with Madelung-LaunoisBensaude syndrome (or Brodie syndrome II) which is characterised by symmetric subcutaneous masses of adipose tissue on the neck, shoulders, arms and upper parts of the trunk. ${ }^{1-3}$

Madelung deformity (MD) of the wrist is characterized by a growth disturbance in the volar-ulnar distal radial epiphysis, so it fails to contribute to linear growth of the corresponding border of the radial diaphysis. Unaffected radial and dorsal portions of the growth plate continue to grow. Faster growing, newly formed bone bends toward area of slower growth, causing the articular surface of distal radius to slant in volar and ulnar direction and a dorsally prominent distal ulna. ${ }^{4,5,6}$ One third of cases of MD are transmitted in an autosomal dominant fashion. The condition has a variable expression and $50 \%$ penetrance. MD is bilateral in $50 \%$ of cases and is primarily found in females. Henry and Thorburn classified MD into 4 different etiologic groups: Posttraumatic, Dysplastic, Chromosomal or genetic (Turner syndrome), and Idiopathic or primary. ${ }^{7}$ Symptoms usually begin during adolescence in girls aged 10-14 years. Patients experience increasing deformity and pain in the wrist with decreased range of motion. Range of motion is decreased, with a limitation of supination, dorsiflexion, and radial deviation. Pronation and flexion usually are normal. 6,8,9

Leri-Weill syndrome : an autosomal dominant dyschondrosteosis (a form of mesometric dwarfism with bilateral Madelung deformity), Turner syndrome, Madelung dyschondrosteosis, gonadal dysgenesis, diaphyseal aclasis (hereditary multiple exostosis), achondroplasia, Hurler mucopolysaccharidosis, Ollier disease..$^{9-11}$ In this report, we describe biilateral, predominant right wrist Madelung deformity in a female with no history of wrist injury or pain despite 13 years of healthy life.

\section{Case History:}

A 14 year old girl hailing from patuakhali came to this hospital with complaints of gradual disfigurement of her right distal forearm over last 11 months and on left side for last 3 months. Initially a painless swelling developed on her right distal forearm which gradually increased to deform her forearm also with bowing of distal two third of forearm . There was no functional impairment, pain or paraesthesia. Similar sort of disfiguring process had started on her left side. There was no involvement of the lower limb or any other joint or bone, no pain or morning stiffness. This event was not preceded by any trauma or surgery.

She has no systemic complaints. None of her family member is affected by this condition. She has a normal birth and development history. Her height for age is below $5^{\text {th }}$ percentile but mental growth is normal. Local examination of her right distal forearm revealed prominence of the ulnar end with dorsal displacement and prominence of the radial end with bowing of lower $2 / 3^{\text {rd }}$ of it. Similar sort of finding present on left hand with lesser extent of deformity. There was absence of redness, muscle wasting. On palpation there was no tenderness or raised local temperature. The swellings seemed to be continuation of the distal end of ulna and radius on respective sides. Extension and radial deviation of right

1. Assistant Professor of Medicine, Dhaka Medical College

2. Honorary Medical Officer, Dhaka Medical College

3. Assistant Registrar of Medicine, Dhaka Medical College

4. Professor of Medicine, Dhaka Medical College

Correspondence: Dr. Md. Robed Amin, Assistant Professor of Medicine, Dhaka Medical College, Dhaka, Bangladesh.Apt-C-2 House no-76, Block-F, Road- 5, Banani Dhaka, Bangladesh. e.mail: robedamin@yahoo.com 

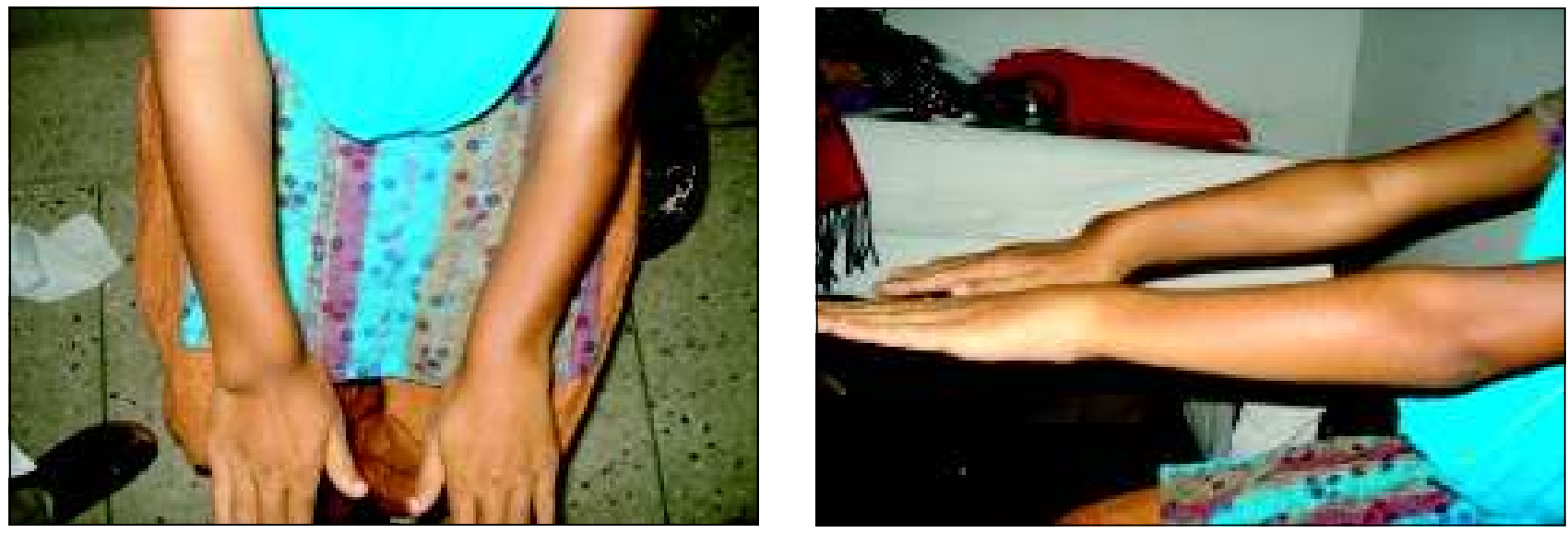

Fig.-1\&2: A swelling at rt wrist joint area and Deformity predominantly in right than left
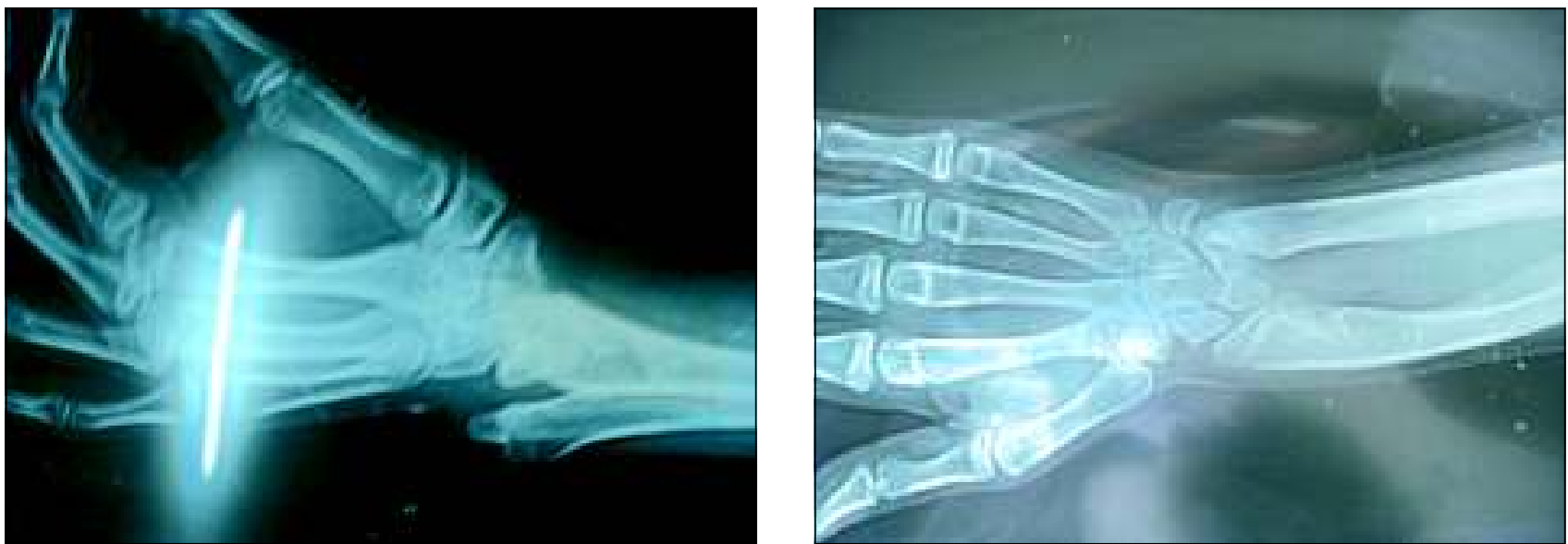

Fig.-2(a \&b) : Dorsal and radial bowing of the radius and exaggerated radial inclination

wrist joint was restricted and in left side flexion was also affected but all movements were painless. Length of forearm and leg was proportional to whole limb. On general and systemic examination there was no other significant finding. A radiological investigation was undertaken in radiology department of Dhaka Medical College hospital. Plain anteriorposterior, lateral, oblique, and closed-fist anterior-posterior radiographs revealed a Madelung deformity with a widened distal radioulnar joint. The findings of radiological examination of wrist and forearm revealed. a) dorsal and radial bowing of the radius. b) exaggerated palmar (up to $35^{\circ}$ ) and ulnar tilt (up to $60^{\circ}$ ) of the radiocarpal articulation. c) failure of ossification of the ulnar side of the distal radial epiphysis.d).exaggerated radial inclination. e) decreased carpal angle below $118^{\circ}$; normal from $118^{\circ}$ to $139^{\circ}$. f) carpal subluxation in a palmar and ulnar direction. $g$ ) lunate is gradually forced to the apex of the V-shaped radioulnaocarpal joint. h)"V-shaped" proximal carpal row with herniated proximal carpal row.i) dorsal subluxation of the distal ulnar and positive ulnar variance. $\mathrm{j}$ ) wedging of the carpus between the radius and ulna.

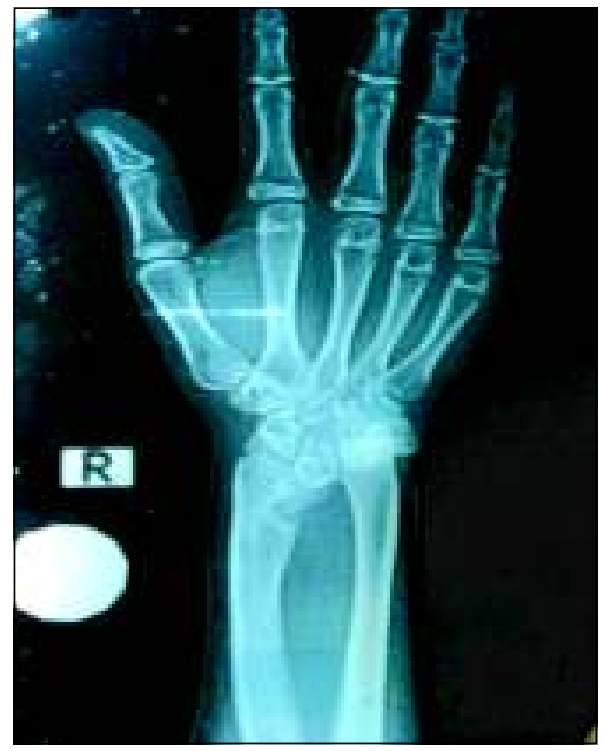

Fig.-3: V-shaped" proximal carpal row with herniated proximal carpal row and dorsal subluxation of the distal ulnar and positive ulnar variance 
She was diagnosed as a case of Madelung deformity of right wrist and initiation of similar deformity in left wrist. Orthopaedics consultation was advised and preparation of surgery was undertaken. But the patients and attendant did not give consent for operative procedure and discharged from hospital with her own deformity.

\section{Discussion:}

The madelung deformity is relatively uncommon, occurring in less than $2 \%$ of the general population, and is most prevalent in female patients, at a ratio of 3 to $5: 1$ compared with male patients. ${ }^{12-16}$ Typically, the deformity is present bilaterally and seldom manifests clinically before the age of 7 years. $9,16,17$ Some authors suggest that the typical middle to late adolescent onset of the disorder may be linked to the adolescent growth spurt. ${ }^{18,19}$

The long-standing and progressive radial deformity gradually worsens until it is suddenly exacerbated by the increased growth rate, often occurring concurrently with a premature physeal fusion. In our case it is also seen that her deformity started just 11 months back while her growth of adolescent started dramatically.

Individuals who present with the disorder are usually limited in pronation and supination of the involved extremity,although the source of that limitation remains controversial. ${ }^{18,19}$ In our case it was also remarkably seen in pronation of wrist and lateral view of forearm revealed the defects clearly visible. The deformity although is easily visible on pronation, it did not revealed any pain or restriction of her daily activities. Possibly this is the reason why she refused to go for corrective operative procedure.

The etiology of the disorder remains unclear, but all hypothetical causes produce a local growth disturbance at the ulnar and volar parts of the distal radial epiphyseal plate. Some have suggested that idiopathic Madelung deformity is probably not a true classifying group but rather a lack of conclusive implication of 1 of the 4 causative groups ${ }^{12,19}$. (Posttraumatic, Dysplastic, Chromosomal or genetic, Idiopathic). Whereas the preceding 3 categories are heritable and not preventable, posttraumatic Madelung deformities are preventable

because the disorder typically develops from long periods of overuse or abuse or both. In our patient there was no such family or genetic background was present and she was also not engaged in a heavy physical work of gymnastic activity. Radiographic findings most often associated with Madelung deformity include increased dorsal and radial bowing of the distal radius, a triangular-shaped carpus, an exaggerated volar and ulnar tilt of the distal articular radial surface, and positive ulnar variance. ${ }^{13,14,15,16,18,19}$ Our patient had all of the characteristics finding of radiology and the diagnosis was done through her clinical history, examination and principally by radiology. Although we did not done the proximal forearm radiology and other joints and lower limb radiology, her clinical presentation was consistent with restriction of disease in wrist area.

Madelung deformity is typically treated with either rest or surgical reconstruction, depending largely on both the specific needs of the athlete and the relative severity of symptoms. Furthermore, surgery is typically discouraged in adolescents and young adults because symptoms tend to subside with age, thus making surgery among the general population necessary only in cases of persistent significant pain. ${ }^{13,19,20}$ In our patients she was asymptomatic but her deformity was rapidly developing and also creates social stigma. That's may be the reason why orthopaedics department wanted to operate on her. Surgical repair usually consists of shortening or resection of the distal ulna or a wedge osteotomy of the distal radius or both. ${ }^{16}$ Epiphysiodesis, corrective osteotomy, and physiolysis in one group, repairs or reconstructions limited to the ulna, including epiphysiodesis, ulnar head excision, shortening osteotomy, distal ulnar resection, and creation of a pseudarthrosis with or without fusion to the radius in the second group and combination of the above techniques in the third group are the surgical options of madelung deformity. Most recent investigators found significant decreases in pain, improved range of motion, and improved grip strength after surgical correction.

\section{Conclusion:}

Madelung deformity is a rare condition that affects the structure and function of the distal radioulnar joint. There are four underlying categories of disorder of this deformity.Although the disorder is commonly linked to several heritable factors, most authors suggest that trauma may also be a factor. Conservative approach in asymptomatic patient is the key but improved surgical technique for symptomatic patients are rewarding now a days.

\section{Conflict of Interest : None}

\section{References:}

1. O. W. Madelung "Die spontane Subluxation der Hand nach Vorne." Verhandlungen der deutschen Gesellschaft für Chirurgie, Berlin, 1878, 7: 259-276.

2. Otto Wilhelm Madelung from whonamedit.com, the dictionary of medical eponyms. Otto Wilhelm Madelung

3. Feliciani C, Amerio P. Madelung's disease: inherited from an ancient Mediterranean population? N Engl J Med $1999 ; 140: 1481$ 
4. Anton JI, Reitz GB, Speigel MB. Madelung's deformity. Ann Surg. 1938;108(3):411-39.

5. Jobe MT, Wright PE. Congenital anomalies of hand. In: Compbell's Operative Orthopedics, 8th Edn. Ed. Crenshaw AH. New York, Mosby Year Book, 1992, pp 3419-3421.

6. Wheeless' Textbook of Orthopaedics, Clifford R. Wheeless, III, MD.

7. Henry A, Thorburn MJ. Madelung's deformity. A clinical and cytogenetic study. J Bone Joint Surg Br. Feb 1967; 49(1): 66-73.

8. Journal of pediatric orthopaedics, October 2001, Volume 10 , Issue 4 Madelung Deformity: Clinical Features, Therapy and Results.

9. Lovell WW, Winter RB, Morrissy RT et-al. Lovell and Winter's pediatric orthopaedics. Lippincott Williams \& Wilkins. (2006) ISBN:0781753589.

10. Lamb D. Madelung deformity. J Hand Surg 1988, 13-1:3-4.

11. Langer LO. Dyschondrosteosis, a heritable bone dysplasia with characteristic roentgenographic features. Am J Roentgenol Radio Therapy 1965, 95: 178-188.

12. Casford B. Madelung's deformity. Available at: http:// brighmrad.harvard.edu /Cases/mcr/hcache/205/full.html. Accessed April 19, 2000.

13. Lamb D. Madelung deformity. J Hand Surg Br. 1988; 13 : 3-4.
14. Leri-Weill dyschondrosteosis. Available at: http:// www3.ncbi.nlm.nih. gov/htbin-post/Omin/dispmim? 127300.cs. Accessed April 19, 2000.

15. UW Radiology Main Online Teaching File. Roentgenographic abnormalitie in Madelung's deformity. Available at: http:// www.rad.washington.edu/ maintf/cases/unk53/answers. html. Accessed April 19, 2000.

16. Brashear HR Jr, Raney RB Sr. Handbook of Orthopaedic Surgery. $10^{\text {th }}$ ed. St Louis, MO: CV Mosby Co; 1986:496497.

17. Dos Reis FB, Katchburian MV, Faloppa F, Albetoni WM, Laredo Filho JJr. Osteotomy of the radius and ulna for the Madelung deformity. J BoneJoint Surg Br. 1997; 80: 817824.

18. Cook PA, Yu JS, Wiand W, et al. Madelung deformity in skeletally immature patients: morphologic assessment using radiography, CT, andMRI. J Comput Assist Tomog. 1996; 20: 505-511.

19. Vickers D, Nielsen G. Madelung deformity: Surgical prophylaxis (physiolysis) during the late growth period by resection of the dyschondrosteosi lesion. J Hand Surg Br. 1992; 17: 401-407.

20. Dawe C, Wynne-Davies R, Fulford GE. Clinical variations in dyschondrosteosis: a report on 13 individuals in 8 families. J Bone Joint Surg Br.1982; 64: 377-381. 